\title{
Assessment of a Public Health Promotion and Education Module at the Graduate Level: A Basis for Revision of Learning Resource Material for Teaching and Training Health Promotion and Education
}

\author{
Jonathan P. Guevarra, Buenalyn Teresita M. Ramos-Mortel, Ernesto R. Gregorio Jr., and Eleanor C. Castillo
}

Department of Health Promotion and Education, College of Public Health, University of the Philippines Manila

\begin{abstract}
Objectives. This study assessed the existing Public Health Promotion and Education 210 module (PHPE 210) as basis for the revision of the learning resource material. Specifically, this study determined the stakeholders' perceptions on the module in terms of: (1) its presentation, organization, topics covered, clarity/user-friendliness and its relevance to practice; (2) its strengths and weaknesses; and (3) the topics that need to be integrated in the resource material.
\end{abstract}

Methods. A qualitative approach was used for this study. Through purposive sampling, 4 focus group discussions and 11 key informant interviews were done. Topic Guide was used as the main data collection instrument. Informed consent was obtained prior to data collection. Data was described verbatim, emerging themes and actual quotations from the participants and informants were extracted in the succeeding analysis.

Results. The results of the focus group discussions and key informant interviews, in general, were similar and actually complemented each other. The module was perceived to be relevant, comprehensive and logically organized. The historical foundation needs to be presented in a more concise and updated manner, and examples and practical applications of the PRECEDE-PROCEED model must be used. To update the module and make it more relevant to the present issues, innovative health promotion strategies such as social media, healthy settings, health in all policies, and multi-sectoral approach must be included in the revised version. Practical applications of the theories and health promotion and education strategies also need to be considered in the revision of the module. The module has to be presented in a more reader-friendly manner, easy-touse and visually appealing to its readers.

Conclusion. This study demonstrated the importance of incorporating the perceptions of educators, module developers, practitioners, experts, and the learners or students in assessing and redesigning a more relevant learning resource material.

Corresponding author: Jonathan P. Guevarra, RN, RM, MAN

Department of Health Promotion and Education

College of Public Health

University of the Philippines Manila

625 Pedro Gil St., Ermita, Manila 1000 Philippines

Telephone: +6325260811

Email: jpguevarra2@up.edu.ph
With the current trends and emerging issues in health promotion and education, an evidence-based development and improvement of learning resource modules to make them relevant to changes have to be continuously undertaken.

Key Words: Health Promotion and Education (HPE), Public Health Promotion and Education (PHPE) Module, module revision

Introduction

The College of Public Health $(\mathrm{CPH})$ is one of the nine degree-granting units of the University of the PhilippinesManila. It is composed of seven departments (health promotion and education; health policy and administration; epidemiology and biostatistics; nutrition; medical microbiology; parasitology; and environmental and occupational health). It offers both undergraduate (Bachelor of Science in Public Health) and graduate degree programs (masters and doctoral degrees). ${ }^{1}$

The Master of Public Health (MPH) program is one of the three flagship programs of the college. The $\mathrm{MPH}$ program requires a student to complete 34 units (core and elective courses) and pass the comprehensive examination in order to graduate and obtain the MPH degree. One of the core courses is Public Health Promotion and Education 210 (PHPE 210) which is offered by the Department of Health Promotion and Education (DHPE). ${ }^{1}$ The Public Health Promotion and Education 210 (PHPE 210) module, referred to as the PHPE Module in this article, is used by the Department of Health Promotion and Education (DHPE) to teach this core course. In addition to the DHPE's primary role of teaching, the department also conducts short courses on basic health promotion and education and on strategies of health promotion and education. The DHPE uses the existing module as reference material in performing its roles in the university.

To strengthen its primary responsibility of teaching in the graduate level, it is imperative for the DHPE to revise the existing module to keep it up to date with current developments and trends in health promotion and education locally and globally. This module will be a useful guide for health promotion and education specialists and practitioners and other health professionals who engage in health promotion and education programs/activities. 
The existing module was first developed in mid-1990 for the Public Health Promotion and Education graduate students. While relevant updates, current trends and issues in the field of health promotion and education were integrated during class discussions, revising the resource manual is still necessary because of the changing context in a globalized world. Health Promotion and Education has been recognized as a major field in public health and its importance in enhancing the quality of life cannot be overemphasized. Local and global developments in the last two decades, as well as the implications of these developments in the practice of health promotion and education, need to be integrated in the said module for it to be an effective instrument in meeting the needs of graduate students and trainees and in keeping up with the demands and challenges of both their present and future roles.

This study assessed the current Public Health Promotion and Education module as basis for revising the resource material. Specifically, this study determined the stakeholders' perceptions on the module in terms of (1) its presentation, organization, topics covered, clarity/userfriendliness, and relevance to practice; (2) the strengths and weaknesses; and (3) the topics that need to be integrated in the resource material.

\section{Materials and Methods}

\section{The PHPE 210 Module}

The first and the original PHPE 210 module was developed by the prime-movers in the field of health promotion and education from the College of Public Health, University of the Philippines-Manila. This module is composed of an introduction and 16 chapters. The introduction presents the definitions of health education and health promotion, its scope, and how the concept of health promotion has evolved over time.

The first three chapters cover the foundations of health promotion and education: the Historical and Philosophical Foundations (Chapter 1), History and Philosophy of Health Education and Promotion in the Philippines (Chapter 2), and Biomedical and Social Science Foundations (Chapter 3). The main teaching strategy in presenting the foundations of health promotion and education is lecture-discussion. Part of the learning experience is a visit to a medical museum to gain a better understanding of the history and evolution of public health and health promotion.

The next three chapters cover the basic processes of learning, communication, and change. Chapter 4 deepens the understanding of the learning process and includes the definition and the elements of the learning process. Selected learning theories (behaviorist, cognitive, humanist theories) are also discussed in this segment. Situations on how people learn, particularly the adult learners, are incorporated at the end of this chapter. Chapter 5 expounds on the communication process, definitions of communication as well as the elements and steps in the communication process. This chapter presents the principles of and barriers to communication, and the different ways to overcome these communication barriers. Some communication theories are also discussed in this segment of the module. Chapter 6 discusses the change process, elements of change, targets of change, barriers and motivation to change. Several models and theories of change are also included in this chapter. Lecture-discussion is used as the main teaching strategy for chapters 4 to 6 and structured learning exercises (SLEs) are also employed when discussing the communication process to encourage active participation. To elucidate the application of the theories and models, the faculty cites specific examples in which they are used.

Chapter 7 provides an overview of the strategies and methods in health promotion and education. The strategies in health promotion and education are presented based on three different ways of classification (according to focus, according to use of behavioral change theories, and according to target factors). Chapters 8 to 15 present the strategies used in health promotion and education, including Counseling (Chapter 8), Group Discussion (Chapter 9), Mass Media (Chapter 10), Social Marketing (Chapter 11), Folk Media (Chapter 12), Enter-Educate Approach (Chapter 13), Training (Chapter 14), and Community Organizing/Social Mobilization (Chapter 15). Each health promotion and education strategy covers the basic concept, principles, and processes behind the use of the strategies. Examples of applications of these strategies are given in the module especially for Mass Media, Folk Media, and Enter-Educate Approach. The strategies and methods in health promotion and education are presented in class by the assigned groups and the faculty provides additional input after the report.

The last segment of the PHPE Module is Chapter 16 which covers Planning and Evaluating the Health Promotion and Education Component of a Health Program. The nine phases of the PRECEDE-PROCEED model (social; epidemiological; behavioral and environmental; educational and organizational; administrative and policy assessments; development and implementation of plan; process evaluation; impact evaluation; and outcome evaluation), which is the main framework used in planning and evaluating health promotion and education programs, are covered in this chapter. Each phase and the steps to accomplish each phase are described in the module. The faculty provides input in terms of concepts, theoretical basis, and the steps involved in planning and evaluating health programs using the framework. A workshop is also conducted to guide the students on the use of the framework in developing a health promotion and education plan. After the workshop, the students finalize their group output for submission to the faculty-in-charge. ${ }^{2}$ 
In summary, the PHPE 210 module is organized into five major segments, namely (a) concepts and principles in health promotion and education; (b) foundations of health promotion and education; (c) processes of learning, communication and change; (d) health promotion and education strategies and methods; and (e) health promotion and education planning and evaluation using the PRECEDEPROCEED model.

\section{Research Design}

A qualitative approach was used for this study. Data was collected through focus group discussions and key informant interviews. A focus group discussion is a qualitative research method which involves a small group of individuals gathered together to discuss their perceptions, feelings, and opinions about a specified topic or issue from which data will be collected. ${ }^{3,4}$ A key informant interview is a qualitative in-depth interview used to gather data from a wide scope of individuals considered target people who have the firsthand knowledge on the specific topic. ${ }^{3}$

\section{Sampling Procedure and Sample}

Purposive sampling was used in selecting respondents for the focus group discussions and key informant interviews. Individuals were selected according to a set of criteria. ${ }^{5}$ Focus group discussions (FGDs) were conducted among graduates of the Master of Public Health program with the Health Promotion and Education track and among sample students who enrolled in the PHPE course during the academic years of 2012-2013 and 2013-2014. Key informant interviews (KIIs) were conducted among faculty members who used the PHPE module, graduates of the Master of Public Health program who are involved in health promotion, experts and practitioners in Health Promotion and Education.

\section{Data Collection and Analysis}

Topic Guide was used as the main data collection instrument for FGDs and KIIs. An informed consent was obtained from the identified focus group participants and key informants prior to data collection. Data was described verbatim, emerging themes and actual quotations from the focus group participants and key informants were extracted in the succeeding analysis. Quotes that were collected in Filipino were translated to English.

\section{Technical and Ethical Approvals}

Technical (CPH Protocol Number 2013-017) and Ethical (UPM-REB 2014-056-01) approvals were obtained from the College of Public Health $(\mathrm{CPH})$ and University of the Philippines Manila-Research Ethics Board (UPM-REB), respectively.

\section{Results}

\section{Profile of Respondents}

This study involved a total of 27 participants from 4 focus group discussions and 11 key informants, 15 of whom are male and 23 are female (Table 1). The FGD participants were selected from current and former graduate students while key informants were former faculty members who used the module as well as selected health promotion practitioners and experts.

Table 1. Distribution of Focus Group Participants and Key Informants based on Sex

\begin{tabular}{lccc}
\hline Sex & Focus Group Participants & Key Informants & Total \\
\hline Male & 13 & 2 & 15 \\
Female & 14 & 9 & 23 \\
Total & 27 & 11 & 38 \\
\hline
\end{tabular}

\section{Focus Group Discussion}

Perceptions on the module in terms of its presentation, organization, topics covered, clarity/user-friendliness, and relevance to practice

In terms of the presentation and organization of the topics of the module, participants unanimously agreed that the topics were arranged logically, chronologically and systematically. The participants appreciated the order of presentation of the topics-the definition of terms and concepts and the foundations of health promotion and education were discussed first to prepare them for understanding the theories as well as the strategies in health promotion and education. One participant commented that: "In terms of sequencing, the topics are presented in a systematic, appropriate manner."

In terms of perceptions on the module's contents/topics, most participants agreed that they were comprehensive and very informative. Moreover, many of the participants stated that all components or topics that they needed were already included in the module's contents. However, they pointed out that some topics were discussed thoroughly and extensively while some were not given the same treatment. It was noted that there was heavy discussion on the history of health education and health promotion and mass media but only a little discussion on the PRECEDE-PROCEED model. There was no discussion at all on the topic of "healthy settings". In as much as some topics were not discussed well, the participants expressed that the depth of discussion was lacking. In fact, one of the students commented that: "The discussion of PRECEDE-PROCEED Model was too short, and examples of the activities, and its application were not provided. The model was just briefly described in a series of steps." Another participant agreed and added that "the module has a lot of topics, so much so that sometimes students would get lost (in the details) in 
which of these are the most important topics that would be covered in the final examinations. The module is too comprehensive for an introductory course."

A few FGD participants agreed that the module is generally clear and user-friendly. Many of them cited several negative attributes of the module that affect its clarity and user-friendliness. Many reported that they experienced information overload. Furthermore, one participant said that the module was "presented in a very textual and heavy manner which is not visually appealing, with some typographical errors and a non-uniform style or format of writing."

The FGD participants were one in saying that the topics covered by the module were very relevant to their field practice or community work. This module even made them realize that there was a more systematic way of doing their community work. The strategies presented in the module had given them suggestions on how to improve their activities in the field while giving them the assurance that what they had been doing before were actually health promotion and education strategies. One of them said that the PHPE module "helped me come up with a realization of a better and more systematic way of doing community work."

\section{Perceptions on the strengths and weaknesses of the module}

For the strengths, the most common response was the perception that the topics in the module were relevant to public health practice. One participant even said that the module made him realize that health promotion was very helpful in community work and that it could be done more systematically. Most participants found the module topics to be very organized and comprehensive. For example, from theoretical foundations to strategies, as indicated by one participant: "[The module provides] a lot of options and strategies on how to promote health. It is not one-sided or unlike what I knew [about health education] before which was only about posters, lectures, and group discussions. [I realized that] there are many other methods to promote health in the community."

In terms of weaknesses, the responses can be categorized as those related to module content and those pertaining to format or style. The most commonly stated weakness of the module related to content was the use of old and outdated examples, e.g., Menudo and Leah Salonga for the Enter-Educate Approach. Another weakness given was the long and heavy discussion of the historical foundation of health promotion and education. Some felt that the topic on the PRECEDE-PROCEED model lacked practical examples.

Many participants identified the poor layout and format of the module as a weakness. Some also found the module packaging (ring bound) as well as the print quality to be poor while others found the material bulky because of the page size (letter $8 \frac{1}{2}$ and 11 in.) and the one-side printing instead of back-to-back printing.

Topics that need to be integrated in the module

Participants in the focus group discussion were also asked what topics they think should be included in the module. One of the most common suggestions was the use of social media in health promotion and education. Alongside this, the participants also thought that new strategies, policies, and laws related to health promotion and education should be considered. In addition, the participants indicated points to improve the discussion of the PRECEDEPROCEED model such as providing examples, in-depth discussion, and further applications. Some further suggested using the new version of the PRECEDE-PROCEED model. The inclusion of more practical examples useful for fieldwork was also recommended.

\section{Key Informant Interviews}

Perceptions on the module in terms of its presentation, organization, topics covered, clarity/user-friendliness, and relevance to practice

The key informants stated that the module was presented and organized in a logical, progressive, and clear manner. However, they indicated that the organization could further be enhanced. One key informant commented: "The organization of the topics is okay, but there should be a way where the reader can sense which is the main topic as well as the subtopics under it." Another suggested the use of dividers at the end of each main topic "to emphasize that the reader has reached the end of the topic."

The key informants said that the content/topics of the module are comprehensive; however, the presentation was too textual, purely narrative, too theoretical and inadequate in terms of illustrations. They further added that the layout and format was unattractive. In fact, one informant said that "there is a tendency to put almost every good topic in the module but then there is a need to emphasize the topics that the target group really need."

Experts who were interviewed generally observed that the topics covered by the module are enough and complete. They also expressed their satisfaction with the coverage of the contents of the module. Some, however, suggested that the module should include the following topics: advocacy, risk communication, dealing with the media, updates on international conferences on health promotion and local policies or administrative orders affecting health promotion and education, new theories on behavior change and the use of social media. Inclusion of updated case studies, examples, and illustrations were also recommended. The informants seemed to echo the observations of the participants from the FGDs. Most of them said that the module "is too technical and it is too bulky for the students to bring." 
All the informants agreed that the PHPE module is relevant in preparing health education and promotion officers (HEPOs) for field work and eventually for greater challenges in their practice. Some informants said that "there were a lot of things that can be learned from PHPE module specially the strategies." Others added that "most, if not all, of these strategies can be applied in the field" and that "we were able to learn the right term for the strategies that we have been applying in our course of work."

\section{Perceptions on the strengths and weaknesses of the module}

In terms of perceived strengths, informants mentioned that the module was very "comprehensive and logically organized". Some informants found the module to be a good reference material because of its comprehensiveness. Some particularly found the discussion of various strategies and the differentiation of health promotion from health education as one of the strengths of the module. Others found the discussion of the PRECEDE-PROCEED model to be essential.

In terms of weaknesses related to content, several informants found the discussion of the history of health promotion and education to be very lengthy. Most informants mentioned that the module was lacking in terms of the use of new and interactive technology such as social media and video- and tele-conferencing. These updates can be incorporated in some health promotion strategies such as communication and training. The module was found to have inadequate discussion on some global developments such as the use of a multi-sectoral approach in health promotion, social determinants of health, and policy advocacy.

Most informants identified the format as one of the weaknesses of the module. Specifically, the lack of illustrations, too textual presentation, and not being readerfriendly were identified as weaknesses. The informants did not see the module as "handy", saying it is too bulky to carry. Aside from the topics, the participants also suggested improving the general format or layout of the module. One participant said that "the module can be improved by using different fonts, font size, borders and dividers." Another added that "tables/matrices, bullets, and icons can be used in the layout and presentation."

\section{Topics that need to be integrated in the module}

The topics suggested by the informants were similar to those given by the FGD participants. According to informants, examples particularly on the topics covering social media, theories, and strategies should be updated. Furthermore, some informants suggested topics such as Health in All Policies approach, advocacy, risk communication, and ways to conduct focus group discussions, monitoring, and evaluation to be considered in the revision of PHPE Module. In terms of the content of the section on the PRECEDE-PROCEED model, a few suggested using the revised and newer adaptation of the model and discussing it in a step-by-step manner supported by more examples. One of the informants found the history of health promotion and education to have been extensively discussed and suggested revising the presentation of the contents. Others said that adding guide questions at the end of each chapter, an annex for additional readings, and a glossary of terms at the end of the module should also be considered.

Aside from the content of the module, the informants also suggested improving the layout and format of the module by using different font sizes and styles, borders, dividers, and more tables. Others further suggested that adding illustrations, graphics, and pictures may help improve the module.

\section{Discussion}

The results of the focus group discussions and key informant interviews, in general, were similar and actually complemented each other. The participants of the FGD and KIIs perceived the module to be relevant, comprehensive, and logically organized. The current organization of the module can be attributed to the intention of the original module writers which was to provide graduate students with thorough and systematic knowledge about health promotion and education. They also aimed to provide learners with thorough knowledge on how health education has evolved over time, the various philosophical, biomedical, and social science foundations of health promotion, its basic processes, an overview of the strategies used in health promotion and education, and to provide students with a practical resource material on how to plan and evaluate a health promotion and education program using the PRECEDE-PROCEED model.

The study respondents found the discussion of the historical and philosophical foundations of health promotion and education to be both very lengthy and textual. The intention of the writers was to provide a comprehensive historical account of how health promotion and education evolved globally and locally, and therefore it was deemed fitting at that time to begin the discussion from the pastoral or prehistoric stage and the Spanish period. However, there is a need to present a more concise and reader-friendly history of HPE. In addition, there is a need to update the historical foundation taking into account the latest developments in health promotion and education in the last 20 years.

Some participants, despite saying that the module is comprehensive, wanted more information on topics like social media, video- and tele-conferencing, risk communication, dealing with media, advocacy, policy advocacy, training strategies, and the multi-sectoral approach, Health in All Policies approach, healthy settings, and social determinants. However, integrating all these contents might defeat the purpose of the material which is to 
serve as an introductory module in health promotion and education. It should be emphasized that the module is to be used by graduate students, specifically by MPH students, to provide them with an overview of the strategies for behavior change. However, some of these contents can be included in the revision of the PHPE module such as the policies and conferences affecting health promotion and education which can be placed under historical developments; innovative strategies such as social media and video- and teleconferencing under communication strategies; and the latest approaches in health promotion such as the healthy settings, Health in All Policies and multi-sectoral approaches. In addition, there is also a need to update the examples cited in the module since there are many scholarly published materials especially on effective strategies in the field of health promotion and education. For other suggested contents, it must be emphasized that these topics are supposed to be taken up by HPE trackers in their elective courses. Advocacy is thoroughly discussed in Public Health Promotion and Education 202 (PHPE 202) - Health Education for Community Development; ${ }^{6}$ risk communication and dealing with media practitioners are included in Public Health Promotion and Education 204 (PHPE 204) - Communications in Public Health; 7 sociocultural determinants of health are the main contents in Public Health Promotion and Education 212 (PHPE 212) Socio-cultural Determinants of Health; 8 and innovative training strategies are taught in Public Health Promotion and Education 214 (PHPE 214) - Teaching of Preventive Medicine and Public Health. ${ }^{9}$ There is a need to balance the contents of the PHPE module such that it is comprehensive, updated, and appropriate as an introductory course.

Another very important result of this study is on health promotion planning and evaluation using the PRECEDEPROCEED model. Study respondents pointed out that there is a need for thorough explanation, relevant examples, and application of the PRECEDE-PROCEED model. Aside from examples in the local setting, applications of the framework in the community, occupational, school, and healthcare settings presented by Green and Kreuter (2005) in the latest edition of their book, Health Program Planning: An Educational and Ecological Approach, can be used as examples in the revised edition of the module. ${ }^{10}$

The study also revealed that there is a need to improve the format and style of the module to make it more readerfriendly. Adding bullets, headings and subheadings, pictures or graphs, icons, matrices, use of more spaces and dividers, could further improve the presentation of the resource material. Most of the participants also felt that the module is too bulky which could be attributed to the use of ring binder and the size of the page (letter) as well as the manner of reproduction (i.e. printing not back-to-back). In this way, the concerns of the users and readers of the module (the module being too text-heavy, too narrative, and not appealing to the users) will be addressed. Thorough proofreading of the revised module is a must to address the typographical errors in the module mentioned by the respondents

Similar to the manner of assessment conducted in this study, different educational institutions in other countries have their own ways of conducting assessment of learning materials. The University College Dublin (UCD) in Ireland has a module enhancement process whose goal is to understand what is working well and what might need some adjustment in order to facilitate more effective learning for future offerings of the module. At UCD, module enhancement is a responsive and reflective activity based on the assumption that there are many variables at play which have an influence on how a module runs and the feedback and experience of the learners are very important components of the process. ${ }^{11}$ A policy on module evaluation also exists at the De Monfort University of United Kingdom which requires every module to be evaluated by the teaching team at the end of each academic session. This takes into account the achievement rates, student views, comments from external examiners, and the observations of the teaching team which have to be responded to as the module is reviewed and updated. ${ }^{12}$

In the University of Stirling in the United Kingdom, module review is an essential part of the University's internal monitoring and review processes. Feedback from students, external examiners, student staff, consultative committees, and other outcomes are considered in this process. ${ }^{13}$ Module review is one of the three levels of quality assurance processes conducted at the Queen's University Belfast. Module Review is a collective self-evaluation undertaken by the staff responsible for the module, supported by external examiners' reports and student views, and, where appropriate, by colleagues within the school. The responsible staff has the opportunity to reflect on the module delivery and to consider plausible ways of presenting the module for the future. This reflection is then supported by external examiners' reports and student views, and by other colleagues within the school. Any changes in the subject environment or innovations in education which would impact the module are also taken into account. All electronic and printed information relating to the module is amended as appropriate. ${ }^{14}$

The assessment of the PHPE module considered the input of both the faculty members who taught the course as well as the graduate students who took the course from the academic years 2012-2014. It is important to note that input from various groups, such as faculty members and health promotion and education practitioners and experts, was also gathered. In this way, the actual experiences of users (students) and the vast experiences of practitioners are considered in coming up with a more relevant and up-todate resource material in health promotion and education. 
Aside from the input from various sectors, the assessment of the PHPE module is also very timely because the University of the Philippines-Manila is in the process of implementing the outcome-based education (OBE) curriculum. For its part, the College of Public Health, particularly the Department of Health Promotion and Education, has also started looking into its course offerings with the goal of applying the OBE framework in the revision of the HPE courses. In its most recent workshop (July 2015), the DHPE revised its syllabus for PHPE 210, including the course outcomes. The next step is the revision of the course resource material taking into consideration the major findings of this study. Other course syllabi will also be evaluated in order for them to conform with the university's goal of providing quality outcome-based education to its constituents.

\section{Conclusion and Recommendations}

This study was conducted to determine the stakeholders' perceptions of the PHPE module in terms of its presentation, organization of contents/topics, topics covered, clarity/user-friendliness, and relevance to practice. It also sought to determine the perceptions on the strengths and weaknesses of the module and the topics that need to be integrated in the resource material. The module was perceived to be relevant, comprehensive, and logically organized. The presentation of historical foundation has to be shortened yet updated and more examples and applications of the PRECEDE-PROCEED model must be provided. Innovative health promotion strategies such as social media, healthy settings approach, Health in All Policies approach and multi-sectoral approach must be integrated in the revised edition of the module. Practical applications of the theories and strategies must be incorporated in the revision of the module. The module has to be presented in a manner that is more reader-friendly, easy-to-use, and visually appealing to its readers.

In developing and redesigning learning modules, a significant, scientific, engaging, and holistic process is very important. This study demonstrated the important role of educators, module developers, practitioners, experts, and the learners or students in developing a more relevant learning material. With current trends and emerging issues in health promotion and education, evidence-based development and improvement of learning modules to make them relevant to changes has to be continuously undertaken.

In future module review and enhancement activities, quantitative measurements in addition to qualitative methodologies will be an important component of the process. It is also important to craft guidelines or standard operating procedures on the implementation of a Module Review and Enhancement Process (MREP). In this way, enhancement of learning materials will be institutionalized and will be part of the learning materials' quality improvement process.

\section{Acknowledgments}

The authors would like to express their sincerest gratitude to the University of the Philippines (University of the Philippines Research Grant Creative and Research Scholarship Fund) for supporting this study. Likewise, their heartfelt thanks is conveyed to Dr. Gisela PadillaConcepcion, the University of the Philippines Vice President for Academic Affairs, for the support extended to the researchers; the focus group discussion participants and key informants, for their significant inputs and invaluable time; and to Mr. Renerio Q. Cordova Jr., Ms. Maribel G. Oidem and Mr. Yves Miel H. Zuniga, for faithfully fulfilling their roles as research assistants.

\section{References}

1. Catalogue 2007-2010. College of Public Health, University of the Philippines Manila, 2007.

2. Reading Notes in Public Health Promotion and Education 210 - PHPE 210 (n.d), Department of Health Promotion and Education, College of Public Health, University of the Philippines Manila.

3. Ulin PR, Robinson ET, Tolley EE. Qualitative Methods in Public Health A Field Guide for Applied Research. Family Health International. Jossey-Bass, A Wiley Inprint. 2005.

4. Wong LP. Focus group discussion: a tool for health and medical research [Online]. 2009 [cited 2012 March]. Available from http://repository.um.edu.my/53578/1/FGD_a\%20tool\%20for $\% 20$ he alth\%20and\%20medical\%20research.pdf

5. Olive P. Purposive Sampling [Online]. 2006 [cited 2012 March]. Available from. http://srmo.sagepub.com/view/the-sage-dictionary-ofsocial-research-methods/n162.xml

6. Public Health Promotion and Education 202 (PHPE 202) - Health Education for Community Development, Syllabus, AY 2013-2014. Department of Health Promotion and Education, College of Public Health, University of the Philippines Manila.

7. Public Health Promotion and Education 204 (PHPE 204) Communications in Public Health. Syllabus, AY 2013-2014. Department of Health Promotion and Education, College of Public Health University of the Philippines Manila.

8. Public Health Promotion and Education 212 (PHPE 212) - Socio-cultural Determinants. Syllabus, AY 2013-2014. Department of Health Promotion and Education, College of Public Health, University of the Philippines Manila.

9. Public Health Promotion and Education 214 (PHPE 214) - Teaching of Preventive Medicine and Public Health. Syllabus, AY 2013-2014. Department of Health Promotion and Education, College of Public Health, University of the Philippines Manila.

10. Green LW, Kreuter MW. Health Program Planning: An Educational and Ecological Approach. NY: McGraw-Hill Higher Education; 2005.

11. University College Dublin. Module Design and Enhancement Overview [Online]. [cited 2014 November]. Available from http://www.ucd.ie/ teaching/resources/moduledesignenhancement/guidetomoduleenhance mentinucd/

12. De Montfort University. Module Enhancement Plans [Online]. [cited 2014 November]. Available from http://www.dmu.ac.uk/documents/ about-dmu-documents/quality-management-and-policy/academicquality/monitoring/module-evaluation/module-enhancement-guide.pdf

13. University of Stirling. Module Review [Online]. [cited 2014 November]. Available from http://www.stir.ac.uk/academicpolicy/handbook/reviewand-monitoring/

14. Queen's University Belfast. Module Review [Online]. [cited 2014 November] Available from http://www.qub.ac.uk/directorates/ AcademicAffairs/ProgrammeApprovalandReviewIncludingHEReview/ ProgrammeReview/ModuleReview/ 